\title{
DGOR-Jahrestagung Frankfurt 1982
}

Die Jahrestagung 1982 der Deutschen Gesellschaft für Operations Research findet in Frankfurt statt in der Zeit vom

\section{2.-24. September 1982.}

Die Tagung soll über den Stand der Unternehmensforschung in Theorie und Praxis informieren, Anwendern wie Grundlagenforschern neue Anregungen geben und den Gedanken- und Erfahrungsaustausch fördern.

Für das wissenschaftliche Porgramm der Tagung sind Übersichts- und Spezialvorträge, insbesondere aus folgenden Gebieten, vorgesehen:

- OR im Bankwesen, im Versicherungswesen, in der Datenverarbeitung, in der öffentlichen Verwaltung, im Gesundheitswesen, im Umweltschutz.

- Produktionsplanung, Aktivitätsanalyse und Lagerhaltung; Logistik; Investition und Finanzierung; Marketing; Rechnungswesen und OR; Personalplanung; Strategische Unternehmensplanung; Netzplantechnik und Projektmanagement; Instandhaltungs- und Ersatzprobleme.

- Stochastische Entscheidungsprozesse, Bedienungs- und Zuverlässigkeitstheorie.

- Prognoseverfahren; Datenanalyse; Qualitätskontrolle; Statistische Verfahren im Rechnungswesen; Simulation.

- Lineare Optimierung; Nichtlineare Optimierung; Ganzzahlige Optimierung; Graphentheorie; OR-Software; Entscheidungstheorie.

Besonderer Wert wird auf Anwendungsberichte aus der Praxis gelegt. Anmeldungen von Vorträgen (in deutscher oder englischer Sprache) sind bis zum

\section{März 1982}

an den Vorsitzenden des Programmausschusses erbeten mit den folgenden Angaben:

a) Autor: Name, Vorname, Titel (auf jedem Blatt)

b) Institution: Firma/Hochschule, Abteilung/Institut

c) Anschrift: Vollständige Postanschrift mit Tel.-Nr. (auf jedem Blatt!)

d) Titel: Titel des Beitrages bitte hervorheben durch Unterstreichung oder Großbuchstaben

e) Zusammenfassung (lang, 6fach): Diese soll im Umfang von zwei Seiten enthalten: Zielsetzung, methodische Einordnung, verwendetes Modell, Anwendungsbereich, Datenmaterial

f) Zusammenfassung (kurz, 6fach): Etwa 10 Schreibmaschinenzeilen zur Aufnahme in das Programmheft.

Es ist vorgesehen, die angenommenen Tagungsbeiträge im Rahmen eines Sammelbandes zu veröffentlichen.

Programmausschuß: Vorsitzender: Prof. Dr. W. Bühler

Universität Dortmund, Lehrstuhl für Investition und Finanzierung

Postfach 500500

D-4600 Dortmund 50

Tel.: 0231/7 55-38 15

Mitglieder: Prof. Dr. B. Fleischmann, Universität Hamburg

K. P. Schuster, Philips GmbH, Hamburg

Prof. Dr. L. Streitferdt, Universität Frankfurt

Dr. H. Zander, Ruhrkohle AG, Essen

Örtliche Tagungsorganisation: $\quad$ Prof. Dr. D. Ohse

Universität Frankfurt, Fachbereich Ökonomie

Postfach 111932

D-6000 Frankfurt 11

Tel.: 0611/7 98-39 79

\section{DEUTSCHE GESELLSCHAFT FÜR OPERATIONS RESEARCH (DGOR)}

gez. G. Fandel (Vorsitzender) 